Article

\title{
Il respiro corto dello Spirito nelle mega-chiese, ai tempi della pandemia
}

\author{
ENZO PACE ${ }^{1}$
}

\begin{abstract}
Riassunto. Che effetti ha la pandemia sulle Mega-chiese? La chiusura forzata o la riduzione drastica dei presenti ammessi ai servizi religiosi ha messo di fatto in discussione sia il regime di verità che molte di queste chiese seguono, dal punto di vista teologico e spirituale, sia la dramma-liturgia del corpo a corpo fra la forza trasmormativa dello Spirito e il principe di tutti i mali, Satana. Si è passati, in tal modo, dall'evento di massa vissuto nei grandi auditori ad un servizio online, a misura domestica, per tanti anonimi e lontani fedeli, cui far giungere un messaggio consolatorio in una fase di disagi e sofferenze nella loro vita quotidiana. L'epidemia ha sottratto la scena ai grandi performer delle Mega-chiese e a quanti, entusiasti, prendevano posto in platea o si muovevano liberamente posseduti dagli spiriti in attesa di esserne liberati, partecipando attivamente al rito liberatorio. Questi fedeli, probabilmente, ora seguono a distan$\mathrm{za}$, da casa, davanti allo schermo del loro computer un'altra scena, meno coinvolgente e, soprattutto, senza la parresia del regime della verità, che si materializzava nello spazio rituale. L'epidemia rappresenta per le mega-chiese una doppia contingenza: da un lato, indebolisce la visione teologica di un Dio che tutto può e dello Spirito che soffia trionfante e sconfigge ogni male, dall'altro, smaterializza la presenza del nemico che diventa invisibile e invadente, non più dominabile fisicamente, da cui nessun leader carismatico è più in grado di liberare i fedeli.
\end{abstract}

\begin{abstract}
What effect has the pandemic on Mega-churches? The forced closure or drastic reduction of those present admitted to religious services has in fact called into question both the regime of truth that many of these churches follow, from the theological and spiritual point of view, and the drama-liturgy of hand-to-hand combat between the transmormative force of the Spirit and the prince of all evils, Satan. In this way, Mega-churches moved from the mass event experienced in large auditoriums to an online service, on a domestic scale, for many anonymous and distant faithful, to which a consoling message can be conveyed in a phase of inconvenience and suffering in their daily life. The epidemic has stolen the scene from the great performers of the Mega-churches and from those who, enthusiastic, took their seats in the stalls or moved freely possessed by spirits waiting to be freed, actively participating in the deliverance's rite. These faithful, probably, now watching from a distance at home, in front of their computer screen, another scene, less involving and, above all, without the parrhesia of the regime of truth, which materialized in the ritual space. The epidemic represents a double contingency for the Mega-churches: on the one hand, it weakens the theological vision of a God who can do everything and of the Spirit who blows triumphantly and defeats all evil, on the other, it dematerializes the presence of
\end{abstract}

\footnotetext{
${ }^{1}$ Professore di sociologia delle religioni all’Università di Padova. Email: vincenzo.pace@unipd.it
} 
the enemy who becomes invisible and intrusive, no longer physically dominable, from which no charismatic leader is more able to delivere the faithful.

\section{Introduzione}

La domanda di partenza da cui prende le mosse questo saggio è molto semplice: che effetti ha avuto la pandemia del Covid19 sulle mega-chiese? Una prima sommaria risposta potrebbe essere altrettanto semplice: laddove è stata imposta dalle autorità politiche il blocco delle attività pubbliche, inclusa la partecipazione alle cerimonie religiose, queste chiese hanno dovuto adattarsi all'inedita situazione in cui si sono venute a trovare. In molti casi, la soluzione era a portata di mano. Molte di queste chiese hanno legato, infatti, le loro fortune ai vecchi e nuovi media. Perciò, non è stato difficile trasferire tutte le attività online. I fondatori e i leader delle mega-chiese hanno investito molti denari per costruire grandi auditori pensati per grandi eventi spettacolari di massa da poter registrare e diffonere in più luoghi (multisite) grazie alle moderne tecnologie telematiche. La pandemia, dunque, non ha posto particolari problemia alle mega-chiese già attrezzate da tempo di tali strumenti di comunicazione a distanza. Laddove si sono diffuse nel mondo, la gestione tecnica di questi luoghi di preghiera è affidata spesso a imprese di audiotech e churchsound. Nel 2019, ad esempio, una delle più grandi mega-church degli USA, la Lakewood di Houston (Texas) ha ammodernato tutto l'impianto audio-video per il suo mega auditorio di 16.800 posti sotto la direzione di un tecnico e di un ingegnere del suono per ambienti ecclesiali in collaborazione con una delle più avanzate ditte specializzate in church-sound (fig. 1).

Figura 1. L'auditorio della Lakewood Church di Houston.

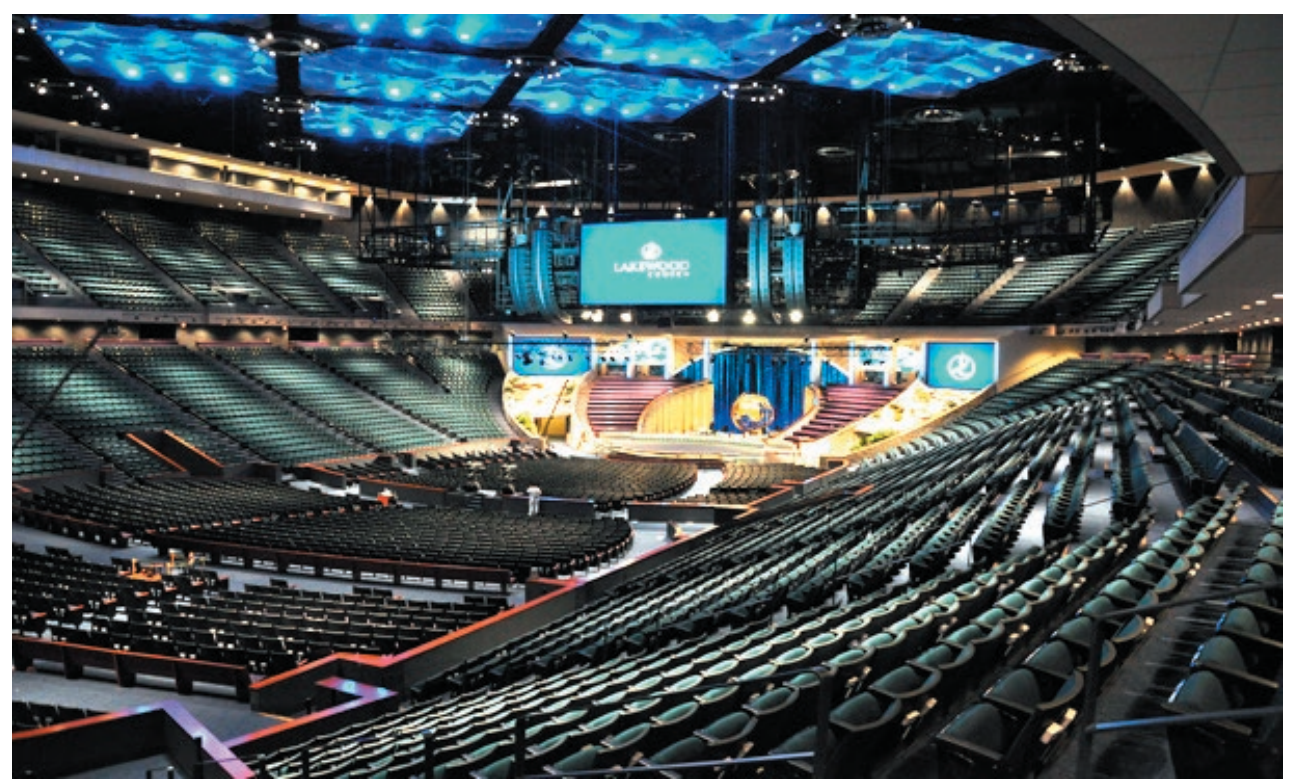


Dal punto di vista, dunque, della comunicazione a distanza, le mega-chiese hanno potuto compensare agevolmente la mancanza di uno spazio fisico. La chiusura forzata ha posto, tuttavia, altri problemi: uno riguarda il regime di verità, per riprendere la terminologia di Foucault (2009) che molte di queste chiese seguono, dal punto di vista teologico e spirituale; l'altro concerne l'azione rituale che viene messa in scena nello spazio fisico, la dramma-liturgia che in molte chiese costituisce il momento centrale di lunghe celebrazioni, in cui si alterano discorsi del leader, sequenze musicali, canti, danze, stati alterati di coscienza, miracoli ed esorcismi. Lanalisi nel presente saggio si concentra su entrambe queste dimensioni.

Dal punto di vista teologico, le mega-chiese di tipo carismastico hanno narrato ai loro fedeli la forza trasformativa dello Spirito Santo, mediata dai poteri carismatici dei leader di tali chiese; tale trasformazione riguarda olisticamente mente, corpo e spirito di un individuo ("tutto è possibile alla forza dello Spirito") e implica non solo un nuovo regime della verità, ma anche la certezza di prosperità materiale, di guarigioni fisiche e di liberazione da spiriti maligni che rendono la vita insopportabile; la forza dello Spirito, perciò, gareggia con le forze del Male, di cui il grande regista è Satana.

Quando l'epidemia ha cominciato a manifestarsi, i leader di molte mega-chiese sono ricorsi al loro lessico familiare: il virus è opera di Satana. Se è così, noi (chiese) siamo abituate a combattere il diavolo e siamo, perciò, in grado di scofiggerlo. Da qui, inizialmente la resistenza e il lavoro di lobby che esse hanno cercato di mettere in campo per costrastare la chiusura imposta per legge dai vari governi nazionali. In un secondo momento, hanno dovuto a malincuore adattarsi alle nuove regole di confinamento. Quando ciò è avvenuto, il repertorio teologico del Vangelo della prosperità e dell'efficacia dello Spirito nello sconfiggere le forze di Satana ha dovuto essere rivisto. Da qui lo spostamento dell'asse simbolico del discorso teologico dal piano dello scontro cosmico fra Dio e Satana a quello più immediato della vita quotidiana dei tanti fedeli più o meno costretti a riguardarsi a casa propria. Si è passati, in tal modo, dal grande evento di massa negli auditori ad un servizio online a misura domestica per tanti anonimi e lontani fedeli, cui far giungere un messaggio consolatorio del tipo "non possiamo condividere l'esperienza straordnaria della presenza reale, fisica dello Spirito, ma possiamo essere certi che esso continua a soffiare dove vuole". Il collegamento via streaming da casa diventa così una sorta di devotional fitness a domicilio, senza più la carica emotiva che un leader sapeva trasmettere dal vivo. Attraverso le emozioni un leader poteva fissare idee (teologiche) e regole (di condotta di vita). Il suo potere carismatico si metteva alla prova, compiendo miracoli o cacciando i demoni in presenza e davanti ad un pubblico emotivamente coinvolto. Il regime di verità, che era affermato (sarebbe meglio dire, in alcuni casi, gridato), nel momento in cui accadevano tali fatti, era che la vittoria sul male era empiricamente dimostrata nella lotta, corpo a corpo, contro Satana che vedeva protagonisti i devoti in preda alla trance. Satana era una figura fisicamente percepibile, da cui ci si poteva liberare sola fide nel potere carismatico del leader-mediatore. La liberazione non era, allora, solo purificazione dell'anima dagli spiriti maligni né tanto meno conversione tutta interiore. Si trattava anche di una liberazione materiale: si guariva da una malattia fisica, ci si emancipava da qualche forma di dipendenza da sostanze tossiche, ci si riconciliava con il partner, ci si serntiva capaci di mobilitare nuove energie per trovare lavoro oppure per avere successo professionale. L'impianto teologico, dunque, di molte formazioni di tipo mega-chiesa che gravitano in un 
quel vasto campo religioso che genericamente è definito del risveglio carismatico è, perciò poco dottrinale, ma molto più fattuale: per governare le persone, il regime di verità di questo modernissimo cristianesimo carismatico, privilegia i fatti e gli atti di fede, non solo il credere, ma il far credere.

Da qui la rivoluzione architettonica e la ristrutturazione dello spazio liturgico che le mega-chiese hanno promosso, marcando le differenze con le Chiese storiche ed anche rispetto al Pentecostalismo storico, che nasce negli USA agli inizi del Novecento. La liturgia non è più concepita come la memoria del sacrificio salvifico di Gesù. Essa è sempre più trasformata in una rappresentazione teatrale, una dramma-liturgia, dello scontro fisico - corpo a corpo, appunto - fra la potenza dello Spirito e le forze del Maligno, sotto la guida sapiente di un leader portatore di poteri carismastici che vengono messi in scena durante le cerimonie pubbliche. Lazione rituale diventa teatro, per riprendere Victor Turner (1982), si configura come la performance di un grande attore che fa muovere sul palco e attorno al palco attori minori in funzione di una messa in scena volta a celebrare le magnifiche sorti dello Spirito che vince, qui ed ora, in tempo reale, la forza di Satana. Lo spazio rituale apparentemente non cambia: la divisione del lavoro sacro fra chi agisce e chi assiste al rito, fra officianti e spettatori, non sembra mutare. Tuttavia, a ben guardare, nelle mega-chiese, al primo colpo d'occhio, si percepisce il cambio di registro simbolico: non cè più un altare (come nella Chiesa cattolica) o uno spazio dedicato per chi guida il culto (secondo la tradizione Riformata) e, in particolare, per chi fa il sermone. Il modello comunicativo è, dunque, quello dell'intrattenimento religioso o della religione da intrattenimento (Pace, 2018), con una precisa finalità: suscitare lo stupore in chi assiste e mostra, a diversi livelli, di volersi far coinvolgere emotivamente da quanto dice e fa il principale attore sul palco. L'uso della voce (dai toni pacati sino a quelli urlati) e dei gesti studiati delle mani, dell'alternanza fra parole e silenzi e dei movimenti (stare fermi, muoversi, cadere in trance in ginocchio e così via) fanno parte di un repertorio drammaturgico che ha la doppia finalità di collocare al centro dell'attenzione l'attore principale - un mattatore, si direbbe, in gergo teatrale - e di avvincere lo spettatore ai suoi poteri carismatici straordinari. I miracoli, gli esorcismi, le profezie e gli oracoli servono (sono mezzi, dunque) a dimostrare che, grazie alla mediazione dei poteri carismatici del leader immediatamente non solo si espellono gli umori maligni dall'anima e dal corpo di un fedele, ma anche e soprattutto si verifica dal vivo, davanti agli occhi di tutti, una sostituzione di sovranità: si caccia un sovrano, Satana, per introneggiarne un altro, lo Spirito divino, una prova empirica di verità, una parresia, direbbe Foucault (2009, p. 160) che si fonda sull'estetica, la mobilitazione e l'eccitamento dei sensi (de Vries, 2008; Meyer, 2009; Da Silva Moreira, 2018) e che mira a disciplinare, attraverso i corpi, le menti.

Cosè accaduto quando la pandemia ha costretto queste chiese a chiudere i loro battenti, a ridurre le performance a numeri piccoli di astanti o, infine, a trasferire le drammaliturgie online?

Dopo aver ricordato brevemente, in una prima parte, qual è la definizione di megachiese che convenzionalmente è largamente accettata nella letteratura sociologica contemporanea, si analizzeranno, rispettivamente nella seconda e terza parte, gli effetti che la pandemia ha avuto sul piano teologico e su quello liturgico.

Tutti i dati sulle mega-chiese sono state raccolti dallautore in occasione di una ricerca sulle chiese di tipo neo-pentecostale condotta in vari momenti rispettivamente in Brasile, 
Ghana, Nigeria e Sud-Africa fra il 2005 e il 2015, utilizzando l'osservazione partecipante, le interviste in profondità a leader e quadri intermedi di tali chiese e l'analisi del discorso, del contenuto e dell'iconografia dei siti ufficiali delle stesse.

Lanalisi su quanto è avvenuto durante la pandemia è stata condotta fra marzo e otttobre 2020, invece, da remoto, visitando le pagine web di queste chiese, partecipando ai riti online e, laddove accessibili, agli spazi interattivi aperti e messi a disposizione per lo scambio di informazioni e comunicazioni all'interno della comunità virtuale.

\section{Le mega-chiese: una definizione provvisoria}

Chi ha la pazienza di entrare nel sito, www.megachurchmyth.com, può trovare non solo la lista aggiornata delle mega-chiese negli Stati Uniti d'America, ma anche visitarle virtualmente una per una. L'elenco è accuratamente tenuto aggiornato da un gruppo di ricercatori dell'Hatford Institute for Religion Research (Connecticut), diretto da Scott Thumma (Thumma, Travis \& Warren, 2007). L'interesse per tale fenomeno nasce da un interrogativo: si tratta di una nuova forma di cristianesimo o semplicemente di unoperazione promozionale, dettata dall'esigenza di presentare il messaggio cristiano in modo più accattivante ad un pubblico di potenziali fedeli, distratti o tiepidi nei confronti delle loro chiese di nascita? Si tratta di un cambiamento profondo oppure di un'operazione di mercato religioso, fatta per competere con un'ampia e sempre più differenziata offerta di beni di salvezza, secondo la scuola di pensiero della rational choice theory (Stark \&Bainbridge, 1991; Young, 1997; Lehman, 2010)?

Sarebbe riduttivo pensare che si tratti solo di una strategia di marketing religioso per attirare fedeli in fuga dalle chiese storiche. Cè qualcosa di più. Le mega-chiese sembrano piuttosto una risposta esteticamente orientata al mercato dei beni di salvezza, ma anche una proposta eticamente rivolta a intercettare un modo di credere proprio degli individui moderni. Per questi individui - in tal senso e solo in tal senso - post-secolarizzati credere è diventato sempre più una scelta e appartenere ad una comunità di fede non ha più i confini fissi e stabili di una parrocchia o delle chiesa di prossimità, in cui si è nati, cresciuti e accanto a cui si vive. Un modo più libero di credere che comporta una più elevata mobilità nel credere (Davie, 1994; Hervieu-Léger, 1999; Diotallevi, 2017).

È bene precisare subito che le orgini del fenomeno delle mega-chiese sono da ricercare nel mondo protestante nord-americano, da anni in rapida trasformazione. Gli edifici di culto tradizionali da un po' di tempo non si riempiono. Predicare il Vangelo durante il servizio domenicale a pochi presenti ha, da tempo, spinto, pastori intraprendenti, dapprima, a indossare i panni del telepredicatore e, poi, a chiudere i battenti delle loro chiese, trasferendosi agli inizi del 2000 nei vasti spazi del web, come nel caso della The First Church of Cyberspace (http://www.godweb.org/sact.html), aperta online da un pastore e teologo di New York, Charles Henderson (Pace, 2013).

I criteri che gli studiosi dell'Hartford Institute hanno convenzionalmente fissato per classificare il fenoneno di cui parliamo, possono essere così riassunti: spazi da duemila posti in su, con una forma architettonica ad auditorio, che cosente di esprimersi da parte del leader con uno stile comunicativo proprio dell'one-man-show.

Quando si parla di mega-chiesa, dunque, il primo riferimento è alla dimensione quantitativa, calcolata dal numero di posti a sedere: dalle 2000 in su. Come dire: più sia- 
mo, più siamo sicuri che il soffio dello Spirito soffi a nostro favore. Parliamo del numero di quanti frequentano mediamente una cerimonia. In base ad esso si può misurare la crescita nel tempo di una chiesa: se in dieci anni, ad esempio, ha dovuto ampliare lo spazio e di quanto; tutto ciò è un semplice indicatore di successo crescente. Nel 1980 negli USA, ad esempio, si contavano 150 mega-chiese; raddoppiano dieci anni dopo (310). Nel 2000 passano a 600 e cinque anni dopo a 1210. Dopo un breve rallentamento fra il 2000 e il 2007 (in crescita di sole quaranta unità), riprende a salire e l'ultima rilevazione del 2014 indica una cifra totale di 1650 unità. La dimensione quantitiva, tuttavia, non ci fornisce informazioni sicure né sul tasso di fedeltà di chi le frequenta né sul sentimento di appartenenza a tali chiese. Si può frequentarle senza doversi sentire necessariamente parte integrante di queste aggregazioni di massa. Una persona può andare ad un servizio offerto da una chiesa per un certo tempo, poi cambiare o smettere del tutto di frequentare qualsiasi altro luogo di culto.

Sinora parliamo di contenitori e non di contenuti. Il contenitore è, tuttavia, di per sé il segno di un cambiamento avvenuto anche nei contenuti. Lattrazione che le mega-chiese esercitano, spostando verso di loro milioni di persone dalle chiese tradizionali dipende, in prima istanza, dai loro leader, spesso ex-pastori di matrice protestante che si sono "messi in proprio", reiventando il loro stile comunicativo, l'organizzazione del rito, i contenuti della predicazione, le forme di partecipazione e, soprattutto, esaltando il loro potere carismatico. Lesercizio del carisma come moderna impresa religiosa tende, dagli USA (Hadaway \& Marler, 2005; Barna, 2006), dove ha iniziato a prendere forma, ad alcuni Paesi dell'America Latina, dell'Africa sub-sahariana e dell'Asia (e più recentemente del Vecchio Continente: dalla Francia all'Ucraina) a concepire lo spazio rituale come luogo di grandi dimensioni adeguate per un grande spettacolo di massa. La preferenza per un modello spaziale per il culto come un auditorio è congeniale per combinare spettacolo e liturgia, intrattenimento e preghiera, partecipazione di massa e emozioni individuali, un momentaneo spazio d'intensità spirituale sospeso nelle anonime aree suburbane, delle grandi metropoli (da Houston a Seul, da Lagos ad Accra, da San Paolo a Santiago del Cile), un luogo in un nonluogo (Augé, 1992). nel senso che non appare più come un luogo familiare, come poteva essere una chiesa architettonicamente coerente con i canoni sacri.

La forma del contenitore riflette e modella i contenuti dellofferta religiosa delle grandi-chiese. L'influenza maggiore si esprime, in particolare, sullo stile comunicativo del pastore che guida il culto; sulla scenografia e coreografia, con un ricorso alla musica-pop cristiana e al multimediale (soprattutto a supporto del sermone o di ciò che continua a somigliare ad esso); sulla riconfigurazione della sequenza liturgica tradizionale (lettura del Vecchio e Nuovo Testamento, Sermone, Comunione, ad esempio), con innovazioni, a volte, profonde. Ad esempio, al posto della lettura dei testi sacri, un display mostra un versetto del Vangelo della domenica senza che il passo da cui tale versetto è tratto sia letto integralmente; oppure al posto della musica sacra tradizionale, un complesso pop o rock cristiano intrattiene vivacemente la platea dei fedeli; o ancora, invece di un altare posto al centro dell'azione liturgica, sovente cè un palco la cui scena ha poco a che fare con segni e simboli sacri.

Non tutte le mega-chiese sono eguali. Occore distinguere fra grandi-chiese che fanno riferimento a una delle tante storiche denominazione protestanti da quelle che, invece, sono nate fuori di esse, per spirito di intrapresa di ex-pastori di questa o quella chiesa e 
da quelle, infine, che, in modo più deciso, si rifanno al risveglio pentecostale che ha avuto inizio nel mondo protestante agli inizi del secolo scorso e poi nelle successive ondate (Cox, 1994; Martin 1990, 2002; Introvigne, 1996; Freston, 2002; Corten, Dozon \& Oro, 2003; Anderson, 2004; Anderson \& Tang, 2005; Pace \& Butticci, 2010; Adogame, 2011). Anche in quest'ultimo caso, tuttavia, non tutte le chiese pentecostali hanno assunto la forma delle mega-chiese; alcune sì, altre sono rimaste gelosamente fedeli all'idea originaria degli inizi del Novecento di dare vita a congregazioni fraterne, senza gerarchie e senza gigatismi architettonici. La forma mega-chiesa si è affermata, invece, con maggiore frequenza in ciò che convenzionalmente chiamiamo neo-pentecostalismo, che emerge a partire alla fine degli anni Settanta e si caratterizza, in molti casi, per l'abbandono del modello congregazionalista del primo pentecostalismo a favore del modello impresa carismatica, un tipo di organizzazione religiosa orientata ad un mercato di beni di salvezza, sempre più differenziato e competitivo (Pace, 2017).

Chi entra per la prima volta nell'auditorio di tali chiese o in una mega prayer city ${ }^{2}$ può avere l'impressione che la struttura stessa sia il messaggio. Una persona di educazione protestante è abituata a pensare o a funzioni religiose solenni, composte, accompagnate dal suono dell'organo e dal canto dei salmi oppure alle più vibranti e coinvolgenti cerimonie delle chiese battiste e pentecostali, può rimanere perplesso nel vedere il ricorso al multimediale nel corso del rito stesso, che rimanda, oltre la voce tonante e a volte urlante del leader, le immagini di una folla che si agita nella preghiera, intensamente partecipata al suono di una musica pop o rock cristiana sparata a dB (decibel) elevati.

È su quest'ultimo segmento particolare del campo religioso occupato oggi dalle megachiese che intendo concentrare l'analisi dell'impatto del covid19. Le grandi chiese che tendono a funzionare come grandi imprese carismatiche, ormai divenute anche transnazionali grazie sia all'azione missionaria sia ai flussi migratori, sono, in teoria, quelle più esposte ai contraccolpi organizzativi che la pandemia provoca. Abituate a chiamare a raccolta migliaia di persone nei grandi auditori, non possono più facilmente contare sulla presenza di massa né investire sul prodigioso manifestarsi dei carismi del leader né, ancor più, sulle possibilità offerte ai tanti che lo desiderano di liberarsi dagli spiriti maligni invocando la forza dello Spirito. Lo Spirito è a corto di fiato?

\section{II Covid19, un complotto di Satana?}

La pandemia ha messo alla prova due dimensioni di queste organizzazioni religiose: la teologia dello Spirito vittorioso sulle strategie malefiche di Satana, da un lato, e la messa in scena dello scontro fisico fra il credente e il principe delle tenebre nello spazio rituale, dall'altro.

Per inquadrare il primo tema, quello demonologico, che fonda il regime di verità che i leader di queste chiese affermano con forza (tutto è possibile a Dio e alla forza dello Spirito), distiguiamo le diverse posture teologiche che tali leader hanno assunto nei vari

\footnotetext{
${ }^{2}$ Più grande di una mega-chiesa, perché, in alcuni casi, può ospitare dalle centomila persone in su non più in uno spazio chiuso, ma in spazi semi-aperti, protetti solo da teloni o da tettoie di eternit, come ho visto in Nigeria fra Lagos e Ibadan, ad esempio, dove a metà cammino si fronteggiano una a destra e una a sinistra, rispettivamente la Redeemed Church, da un lato, e la Mountain of Fire and Miracles, dall'altro
} 
momenti in cui il Covid19 si è manifestato: dall'inizio (gennaio-febbraio 2020) al moltiplicarsi dei focolai d'infezione in varie parti del pianeta sino al riconoscimento ufficiale da parte dell'OMS della pandemia il 13 marzo 2020 e, infine, ai provvedimenti presi dai governi di chiusura di tutti i luoghi pubblici (compresi i luoghi di culto), soprattutto nella fase più acuta di contagio diffuso fra marzo e giugno 2020. La seconda fase autunnale, da questo punto di vista, non ha mutato di molto lo scenario.

Innanzitutto, si può constatare con un rapido controllo via web delle attività delle principali mega-chiese che, in generale, esse si sono adeguate alle regole sanitarie restrittive, salvo un piccolo numero di queste che, invece, hanno cercato, di fronte al primo lockdown di resistere allordine di chiusura dei luoghi di culto, ricorrendo a due argomenti. Il primo suonava pressappoco così: "il Covid19 è opera di Satana, noi abbiamo le armi per combatterlo e, conseguentemente, per sconfiggere questo virus, che sembra essere né più né meno che una variante dell'influenza". Il secondo consequenziale argomento è stato: la preghiera è un'arma spirituale efficace per batterlo. I leader carismatici di queste megachiese si sono presentati come i garanti di una sicura vittoria sulle forze del male. Tale primordiale postura teologica ha subito, in molti casi, clamorose smentite dalla dura realtà dei fatti: alcuni leader si sono ammalati (anche gravemente) di Covid19; in altri casi, i luoghi di culto, affollati e occupati sino all'inverosimale dai fedeli, sono diventati veri e propri focolai d'infezione (in alcuni casi, il primo focolaio, come in Corea del Sud e in Francia); infine, la convizione che il virus non fosse altro che una variante dell'influenza stagionale, in sintonia con quanto affermavaano, del resto, alcuni leader politici ha ceduto il passo ai timori sempre più diffusi fra la popolazione della contagiosità e della pericolosità del virus, con la coseguenza che anche queste mega-chiese si sono acconciate allordine di chiudure i loro luoghi di culto.

Venendo a mancare la demonologia con funzioni sanitarie pubbliche che queste chiese invocavano per affermarsi in un campo religioso differenziato e competitivo, dove altre chiese avevano, invece, preso sul serio le preoccupazioni e prescrizioni dei medici e degli scienziati senza evocare scenari apocalittici, il discorso si è spostato sulla funzione civile che le mega-chiese hanno rivendicato come fornitori di servizi essenziali alla popolazione (Bandeira \& Carranza, 2020; Oro, 2020). Alcuni esempi concreti aiutano a comprendere meglio quale sia il repertorio teologico o, in termini più neutri, l'agire comunicativo di alcune mega-organizzazioni religiose.

In Brasile un gruppo di mega-chiese è oggi in parte ben rappresentato anche in Parlamento, formando ciò che viene chiamata la Bancada evangélica o Frente Parlamantar Evangelica. Si tratta di un cartello che riunisce vari deputati e senatori ${ }^{3}$, militanti prevalentemente in due distinti partiti di centro-destra, affiliati ad alcune delle più rilevanti organizzazioni religiose di area pentecostale e neo-pentecostale. ${ }^{4}$ A tenere assieme politicamente personaggi di spicco di varie mega-chiese è un programma di lotta contro l'aborto, l'eutanasia, le unioni civili fra persone dello stesso sesso, così come lopposizione a qualsiasi forma di riconoscimento di diritti civili alle persone omosessuali e transessuali, considerate affette da disturbi mentali. Il nuovo Presidente Jair Messias Bolsonaro, eletto grazie

\footnotetext{
${ }^{3}$ In occasione delle elezioni della $56^{\wedge}$ legislatura, svoltesi nel 2019, questo cartello riuniva 195 deputati e 8 senatori. Nel 2014 erano rispettivamente 87 e 3.

${ }^{4}$ In particolare, le più rappresentate sono rispettivamente le Assemblee di Dio, la Igreja Universal do Reino de Deus, la Igreja Internacional da Graça de Deus e la Igreja Batista.
} 
al voto massiccio di fedeli appartenenti alle grandi chiese pentecostali e neo-pentecostali, incarna politicamente l'idea del Brasile nazione cristiana, redenta dai mali prodotti dalla sinistra socialista, durante gli anni dell'egemonia del Partito dei Lavoratori guidato da Lula (Alves, 2018; Almeida, 2019; Mariano \& Gerardi, 2019; Prandi, Dos Santos \& Bonado, 2019). Bolsonaro, del resto, durante la campagna elettorale, aveva manifestato più volte la vicinanza con il mondo pentecostale e neo-pentecostale del suo Paese, accreditandosi come il salvatore della patria. ${ }^{5} \mathrm{Si}$ comprende, allora, perché, quando scoppia la pandemia in Brasile, l'allenza politico-religiosa che Bolsonaro aveva realizzato con una parte delle mega-chiese di cui parliamo è stata subito messa alla prova. In un primo tempo, infatti, mentre il Presidente si mostrava scettico nei confronti dell'allarme che proveniva dal mondo medico-scientifico sulla gravità del contagio provocato dal virus, alcuni dei leader religiosi più popolari levavano la loro voce per dire che il malanno non era altro che opera di Satana e che le sue arti malefiche potevano essere sconfitte invocando la forza dello Spirito. È la posizione assunta, ad esempio, da Edir Macedo, il fondatore e leader della Igreja Universal do Reino de Deus, una delle più estese mega-chiese in Brasile e in altri Continenti, all'inizio della pandemia. Un altro influente leader pentecostale, Salas Malafia, in occasione di un grande raduno dei suoi fedeli a Brasilia, alla presenza del presidente Bolsonaro, aveva evocato il virus come lo Spirito di morte, che, dunque, poteva essere battuto invocando lo Spirito della vita, lasciandosi andare a considerazioni parascietifiche del tipo: "il DNA dei brasiliani ci rende immuni nei confronti del coronavirus19". Un altro pastore neo-pentecostale, Joao Pessoa dell'Associazione Fé Perfeita, ha profetizzato, a sua volta, una rapida negativizzazione delle persone che credono in Cristo: "il diavolo se ne tornerà a casa presto con le pive nel sacco!".

Atteggiamenti simili a questi appena ricordati sono stati condivisi da altri leader di mega-chiese in altre parti del mondo. In molti Paesi dell'Africa sub-sahariana, dove sono nate e fioriscono mega-chiese neo-pentecostali, l'evocazione della figura del demonio responsabile della pandemia rappresenta un motivo ricorrente nei repertori retorici che i leader carismatici utilizzano per convincere i loro seguaci che sono in guerra spirituale (spiritual werfare) con Satana. L'unica risposta, perciò, per loro non è la chiusura dei luoghi di culto, ma la moltiplicazione delle occasioni per grandi raduni in cui, anche fisicamente, si manifesta la volontà di combattere contro le arti del demonio: fighting for Jesus. In Zimbabwe, Emmanuel Makadiwa, fondatore della United Family of International Church, auto-proclamatosi profeta ha sostenuto alla fine di febbraio 2020 di aver già nel 2016 previsto l'arrivo di una pandemia che avrebbe causato migliaia di morti, aggiungendo che essa non è altro che opera del demonio. Il mese successivo, dopo aver ribadito che l'unica medicina contro il coronavirus è dare ascolto alla voce del profeta (cioè lui, che parla in nome di Dio), ha aggiunto che Dio gli ha rivelato che la medicina più adatta è la clorochina (la pillola anti-malarica), mostrando in un video che anche Trump negli USA e Modi in India avevano consigliato questa cura. Più di recente, Makadiwa ha sposato la tesi del complotto (sempre ordito da Satana): l'an-

\footnotetext{
${ }^{5}$ Uno dei segni più clamorosi è stato il battesimo nelle acque del Giordano celebrato da un pastore delle Assemblee di Dio nella primavera del 2016, da Everardo Dias Pereira, candidato alla presidenza del Brasile alle elezioni 2014 e presidente del Christian Social Party (PSC). Bolsonaro, che non ho mai rinnegato le sue origini cattoliche, ha dichiarato di aver frequentato per dieci anni la Chiesa battista (cui aderisce tuttora sua moglie Michelle) prima di rinnovare la promessa battesimale nelle acque del Giordano sotto la guida spirituale di un pastore pentecostale.
} 
nuncio dell'imminente vaccino è, secondo lui, un'ulteriore macchinazione mondiale del demonio; quando sarà iniettato, il siero veicolerà un microchip sottocutaneo a milioni di persone, che saranno così controllate meglio dal Signore del male. Altri leader di mega-chiese nigeriane e ghaneane hanno cercato di intepretare il complotto ordito da Satana in vario modo, cercando di declinare il tema a seconda delle specifiche retoriche elaborate da ciascuno di loro nella competizione elevata fra tali chiese. Ad esempio, TB Joshua, il leader della nigeriana Synagogue Church for all Nations agli inizi di marzo aveva previsto che il virus sarebbe stato sconfitto il 27 marzo $^{6}$. Il capo della Mountain of Holy Ghost Intervention Church, il nigeriano Odumeje, finché i luoghi di culto sono rimasti aperti (sino a metà marzo), ha accentuato tutte le sua performance abituali di esorcismo e miracoli, rappresentandole come verie e proprie sedute di wrestling, la lotta corpo a corpo con il demonio. Oyakhilome, a sua volta, fondatore della Christ Embassy, che ha la casa madre a Lagos, è arrivato a dire che il coronavirus è la tattica usata da Satana per sottomettere i popoli al dominio di un nuovo ordine geopolitico voluto dalle cinque potenze più industrializzate del mondo.

Analoghe situazioni le troviamo anche in Corea del Sud, un altro grande laboratorio socio-religioso a cielo aperto nel mondo contemporaneo, dove fioriscono nuove chiese di ispirazione cristiana e alcune di queste assumono la forma della mega-chiesa. Due di queste sono state accusate di essere state focolai del coronavirus nel marzo 2020. Si tratta, rispettivamente, della Shincheonji (letteralmente: Il Tempio del Testimone) e della Sarang Jail (letteralmente: Lamore viene prima di tutto). La prima è stata fondata nel 1961 nella città di Daegu da Lee Man-hee, proclamatosi messia (o promised pastor) giacché egli incarnerebbe lo spirito di Cristo e, dunque, sarebbbe solo lui capace di leggere i segni dei tempi dell'imminente secondo avvento di Gesù ${ }^{7}$. In una delle assemblee ai primi di marzo la sua chiesa è stata uno dei primi focolai d'infezione con 312 casi, che, a loro volta, hanno infettato più di cinquemila persone. Per questo Lee è stato arrestato; a nulla è servito il suo pubblico gesto di pentimento (in ginocchio con la testa sul pavimento). La retorica anti-virus, fino ad allora, era che esso fosse opera del maligno, una prova di fede per i veri credenti: i pochi eletti che continuavano a sfidare le arti di Satana radunandosi in intense preghiere attorno al messia sarebbero stati immuni dal contagio.

La seconda mega-chiesa (Sarang Jeil) è nata nel 1956 per impulso di Jun Kwang-hoon. Nei suoi discorsi il tema del complotto demoniaco è illustrato ricorrendo a due registri: l'epidemia è l'occasione per promuovere la rinascita spirituale del popolo coreano fiaccato dal consumismo e dal materialismo e, così facendo, per sconfiggere il disegno politico dell'attuale Presidente coreano (eletto nel 2017) Moon Jae-in ${ }^{8}$ accusato di voler imporre un regime comunista per giungere poi alla riconciliazione con la Corea del Nord. Anche la chiesa di Jun è stata uno di focolai del virus con 676 casi, incluso il leader stesso.

\footnotetext{
${ }^{6}$ Passata questa data, a chi gli rimproverava di essersi sbagliato e di aver diffuso false notizie, Joshua si è difeso dicendo che in effetti egli si riferiva alla fine dell'epidemia a Wuhan! TB Joshua si è sbilanciato a fine maggio sulle elezioni americane, quando Trump ha nominato come giudice della Corte Suprema Amy Coney Barrett: la sua nomina, egli ha detto, è un segno di Dio, Trump si appresta a vincere le elezioni!

${ }^{7}$ Lee Man-hee è stato anche un esponente politico del Christian Liberal Party.

${ }^{8}$ E il secondo Presidente cattolico nella storia repubblicana della Corea del Sud, figlio di una famiglia di rifugiati nordcoreani, fa parte del Partito Democratico Unito di orientamento liberale (e non certo di sinistra). Nell'aprile del 2018 è stato il primo Presidente a varcare il confine con la Corea del Nord nella zona smilitarizzata e, cinque mesi dopo, a far visita a Pyongyang su invito di Kim Jong-un.
} 
Potremmo moltiplicare gli esempi: in Nuova Zelanda la Destiny Church guidata dal pastore Brian Tomacki nei suoi sermoni ha ripetutamente sostenuto che il demonio si è impossessato del virus aumentandone la carica virale e che il confinamento e la necessaria chiusura dei luoghi di culto dovevano essere intepretati come un giusto castigo inviato da Dio per quanti non hanno più fede in Lui. L'idea della punizione divina - l'altra faccia delle strategie malefiche di Satana - nutre, d'altro canto, i discorsi apocalittici di numerosi pastori evangelicali negli USA e in America Latina. Irvin Baxter, ad esempio, che gestisce una stazione radio e relevisiva molto seguita (la Prophecy Radio e la TBN TV), durante la campagna elettorale a favore di Trump, ha più volte affermato che l'epidemia è una punizione divina per quanti hanno relazioni sessuali prima del matrimonio, giustificano le unioni omosessuali e sono a favore dell'aborto.

L'approccio demonologico al Covid19 non è appannaggio solo dei leader carismastici delle mega-chiese. In alcuni casi, tale atteggiamento è condiviso anche da uomini politici che ricoprono autorevoli cariche istituzionali. Ad esempio, il Presidente del Tanzania, John Pombe Magufuli ha più volte ripertuto, dinnanzi alle prime avvisaglie del contagio (che nel suo Paese, per la verità, è stato molto limitato), che il virus è opera del demonio e che l'unico modo per contrastarlo è affidarsi a Gesù; ha indetto, perciò, grandi raduni di preghiera (sovente con un gran numero di persone poco protette dalla mascherina); ha così potuto dimostrare che solo la preghiera è stata la vera medicina che ha sconfitto il male, voluto da Satana. Anche Bolsonaro in Brasile ha sponsorizzato grandi cerimonie pubbliche: come, ad esempio, in occasione della Pasqua in aprile 2020, una preghiera per la nazione cristiana, invitando tutti i rappresentanti delle maggiori organizzazioni religiose. Infine, la consigliera spirituale di Donald Trump, la predicatrice televangelica e leader dal 2019 della mega-chiesa New Destiny Christian Center (in Apokpa, Florida), Paula WhiteCain, aveva salutato la sua vittoria alle elezioni del 2016 come il segno di Dio per l'avvento del cristianesimo al potere, e che, a suo tempo, aveva contribuito a disegnare la figura del suo avversario, Barack Obama, come il rappresentante di Lucifero in terra ${ }^{9}$.

\section{II rito assente}

Vediamo ora se e come la guerra spirituale che molte mega-chiese hanno predicato e, sino a qualche mese fa, messo in scena nei riti settimanali sia stata compromessa dalla chiusura dei luoghi di culto imposta dai vari governi nazionali e, in particolare, che cosa abbia significato il passaggio dal reale al virtuale per quelle chiese che della dramma-liturgia, della lotta corpo a corpo con Satana, hanno fatto l'elemento rituale caratterizzante e competitivo con altre formazioni religiose.

I luoghi di culto di tutte le principali religioni mondiali sono stati colpiti dalla pandemia, nel senso che sono stati o chiusi oppure severamente controllati e contingentati gli accessi. Le polemiche nei confronti dei governi nazionali che hanno imposto tali restrizioni non sono mancate. Nel caso specifico delle mega-chiese, la forzata sospensione delle liturgie di massa nei grandi auditori è stata compensata, solo in parte, dalla diffusione delle celebrazioni via rete. Per molte, alla necessaria rinuncia alla messa in scena del rapporto

\footnotetext{
${ }^{9}$ Il 4 novembre 2020, all'indomani delle elezioni presidenziali White Cain rassicurò Trump sulla sua rielezione, dichiarandosi sicura che dall'Africa alle Americhe tutti gli angeli sarebbero scesi a fianco di The Donald.
} 
vivo fra un leader carismatico e la folla dei seguaci, i mezzi di comunicazione (vecchi e nuovi), che già queste chiese avevano imparato ad utilizzare da anni per diffondere il loro messaggio e ampliare il culto della personalità del leader, hanno sopperito parzialmente all'esperienza fisica del contatto con la voce, il corpo, la gestualità, le parole, l'agire sul palco dell'one-man-show. I repertori narrativi che prendevano forma attraverso la presenza fisica del leader e diventavano mezzi potenti per trasmettere emozioni e cognizioni, passioni e convinzioni, sono apparsi in rete altra cosa rispetto all'esperienza diretta vissuta nei grandi auditori da miglia e miglia di persone in essi convenuti. Il passaggio dal reale al virtuale delle calorose e accaldate sessioni di liberazione dagli spiriti maligni ha raffreddato inevitabilmente le alte temperature emotive che nelle sedute dal vivo il leader riusciva ad elevare. Nella maggior parte dei casi, l'impeto comunicativo del leader ha dovuto sottostare alle regole di una comunicazione online a distanza, assistita dal computer: l'immagine del leader è apparsa fissa, su uno sfondo privo di ornamenti particolari - salvo il logo della chiesa -, la sua figura si è profilata tanto più immobile e compassata quanto più prima della chiusura dei luoghi di culto era in movimento sul palco, a volte eccessivamente agitato nella voce che saliva di tono o nei gesti concitati o nel corpo percorso da tremiti.

Lanalisi netnografica ${ }^{10}$ delle immagini e dei messaggi delle pagine youtube che le mega-chiese hanno aperto nei loro rispettivi portali ufficiali ci ha mostrato come in assenza di un contatto diretto con una comunità di seguaci, i leader tendono a somigliarsi tutti fra loro: soli sullo schermo del computer, fermi, ingessati e un po' disorientati di fronte al vuoto di una platea invisibile, capaci tutt'al più di modulare la frequenza della voce e di cambiare le espressioni del volto (le immagini dei primi piani non potevano essere alternate alle immagini di gente orante, in preda alla trance o in estasi, rapita dalle parole del leader). Insomma, la solitudine dei leader senza più le folle adoranti è l'immagine prevalente che si può osservare nel web, passando in rassegna un campione di megachiese sparse nei vari continenti, dove si sono diffusi. Laddove, le mega-chiese, assieme ad altre chiese storiche sono riuscite a mantenere aperte i loro luoghi di culto, reclamando la loro funzione di provvedere servizi essenziali alla popolazione ${ }^{11}$, ma in tutta sicurezza (con pochi astanti, ben distanziati e con mascherina), queste chiese hanno potuto trasmettere le cerimonie online, ma senza più la coralità, l'estasi o l'effervescenza collettiva che le caratterizzava prima della pandemia.

Il ritmo coinvolgente delle sessioni di preghiera dal vivo viene, in tal modo, sostiuito da uno stile comunicativo composto e solenne, che tende a spostare l'attenzione di chi si collega, tramite youtube o facebook, verso i contenuti del messaggio (si leggono più salmi o passi dei Vangeli); il pastore o il leader, quando appare, si mostra assorto, raccolto in

\footnotetext{
${ }^{10}$ Si deve a R. Kozinets (2015) il neologismo.

${ }^{11} \mathrm{Ci}$ sono state controversie fra alcune mega-chiese e i governi nazionali. Il caso più vicino a noi riguarda l'Èglise Porte Ouverte Chrétienne di Moulhouse (una mega-chiesa che può ospitare nel suo auditorio abitualmente duemila e trecento persone). Tra la fine febbraio e la prima metà di marzo 2020 il luogo di culto è diventato un focolaio di contagio nella regione dell'Alto-Reno francese e anche in Burkina Faso, dove la chiesa ha una missione. La Chiesa ha dapprima cercato di tenere aperto il luogo di culto, anche con un numero ridotto di fedeli, nonostante le pressioni delle autorità politiche. La situazione è precipitata a fine marzo, quando il pastore della chiesa è stato colpito dal virus. Tutte le cerimonie da quel momento sono passate sulla rete e, in una delle sue prime apparizioni, il pastore, fisicamente provato, ha invitato in modo accorato i fedeli ad attenersi alle misure di sicurezza imposta dall'autorità ed a seguire i riti offerti online (su questa Chiesa: Amiotte-Suchet \& Willaime, 2004; Fath, 2008).
} 
preghiera o ripreso in luoghi isolati in meditazione. Ad esempio, il leader della Mounatin of Fire and Miracles, Daniel Olukoya, inventore della "preghiera violenta" contro Satana e predicatore trascinante, con la diffusione dell'epidemia in Africa, dove la chiesa è maggiormente presente, ha lanciato dagli schermi l'idea delle settimane di preghiera e digiuno per sconfiggere il virus. Non potendo officiare sessioni di esorcismi e di liberazione dagli spiriti maligni in presenza, ha per così dire spiritualizzato il messaggio: alla pratica del corpo a corpo con il demonio incoraggiata durante le sessioni liturgiche, Olukoya ha provato a convertire lo spiritual warfare in una disciplina del corpo e della mente, un'ascesi cui ogni individuo può sottoporsi a distanza ad una sorta di devotional fitness come a le varie sedute di yoga o aerobica via cavo. TB Joshua, invece, ha cercato di mantenere alto il livello di fiducia, che si è guadagnato in tutti questi anni, di guaritore dal vivo, ma anche a distanza (ho visto una fila di persone che gli mostravano foto di persone care malate chiedendo di esercitare a distanza i suoi poteri taumaturgici). Mentre egli, durante il lockdown, si mostra in video in luoghi isolati, asssorti nella preghiera e nella meditazione, recitando salmi e profetizzando la vittoria di Trump o la fine imminente della pandemia, continua, tuttavia, via satellite a collegarsi con persone malate che implorano la sua potenza di guaritore.

Tutte quelle mega-chiese che hanno investito molto sull'ermeneutica vivente della lotta fra la forza dello Spirito e quella del Maligno, facendo della spettacolarizzazione di questa sorta di scontro di civiltà (la città di Dio vs. la città del Demonio) celebrato nelle prayercity, hanno, in alcuni casi, come abbiamo visto dal Brasile alla Corea del Sud, dagli USA allo Zimbabwe, hanno altresì cercato di accreditarsi come chiese-salvatrici della nazione. Lepidemia per queste chiese è la levatrice di società redente che tornano a proclamarsi città di Dio.

\section{Conclusione}

L'epidemia ha sottratto la scena ai grandi performer delle mega.chiese e a quanti, entusiasti, prendevano posto in platea o si muovevano liberamente posseduti dagli spiriti in attesa di esserne liberati, partecipando attivamente alla dramma-liturgia. Questi fedeli, probabilmente, ora osservano freddamente da casa, davanti allo schermo del loro computer (quando ce l'hanno o hanno voglia di accenderlo) un'altra scena, meno coinvolgente e, soprattutto, senza la parresia del regime della verità, che si materializzava nello spazio rituale. Lo Spirito sembra non soffiare più, ha il respiro corto nel cyber-spazio.

Le ultime osservazioni indicano che siamo di fronte ad un passaggio inatteso nella relativa breve vita delle mega-chiese di tipo carismatico, dovuto all'impatto della pandemia sul modello organizzativo che tali chiese hanno realizzato. Non potendo più contare sulla presenza fisica di tanti fedeli come quelli che accoglievano in auditori sempre più grandi, il rischio di bancarotta finanziaria per alcune di queste chiese è dietro l'angolo. Lo spettacolo dal vivo era anche un modo per stimolare i partecipanti a diventare fedeli, invitandoli a versare denaro (o la decima come nel caso di alcune mega-chiese, come, ad esempio, la Igreja Universal do Reino de Deus brasiliana) o fare donazioni alla Chiesa.

A parte questo dettaglio pratico, ciò che mi interessava mostrare nel presente saggio è la doppia contingenza che il Covid19 rappresenta per il mondo delle mega-chiese: a) il virus indebolisce la visione teologica di un Dio che tutto può e dello Spirito che soffia trionfante e sconfigge ogni male, ma non sembra in grado di farlo nei confronti del virus; 
la teologia della prosperità mostra tutti i suoi limiti, giacché una chiesa può diventare focolaio di contagio nel momento stesso in cui celebra i suoi riti; nemmeno il big man del big God sfugge, a volte, all'infezione; b) il virus fa uscire di scena - dallo spazio drammaliturgico - il demonio, per occupare la scena mondiale, diventa un nemico invisibile con cui è impossibile replicare all'infinito il corpo a corpo individualizzato e materializzato nello spazio rituale che le mega-chiese offrivano ai loro fedeli.

\section{Riferimenti}

Adogame, A. (2011). Who is afraid of the Holy Ghost? Pentecostalism in Africa. Trenton: African World Press.

Almeida, R. (2019). Bolsonaro Presidente. Conservadorismo, evangelismo e a crise brasileira. Novos Estudos Cebrab, 38, pp. 85-213.

Alves, J. (2018). O voto evangéligo garantiu a eleição de Jair Bolsonaro. PortalEcoDebate, 21/10/2 (http://ecodebate.com.br : ultimo accesso il 20 novembre 2020).

Amiotte-Suchet, L., Willaime, J-P. (2004). La pluie de l'Esprit. Paris: Èditions du GSRL.

Anderson, A. (2004). An Introduction to Pentecostalism. Cambridge: Cambridge University Press.

Anderson, A. and Tang, E. (2005). Asian and Pentecostal. Leiden: Brill.

Augé, M. (1992). Nonlieux. Paris : Seuil.

Bandeira, O. and Carranza, B. (2020). Reaction to the Pandemic in Latin America and Brazil: Are

Religions Essential Services?, International Journal of Latin-America Religions, October, (https://doi.org/10.1007/s41603-020-00116-0 : ultimo accesso il 22 ottobre 2020).

Barna, G. (2006). The State of the Church. Ventura: Barna Group.

Corten, A., Dozon, J-P., Oro, A.P. (2003). Les nouveaux conquérants de la foi. Paris: Karthala.

Cox, H. (1994). Fire from Heaven. Boston: Addison-Wesley.

Da Silva Moreira, A. (2018). The Aestheticization of Religion in Brazil. International Journal of Latin American Religions, 2, pp. 125-141.

Davie, G. (1994). Religion in Britain since 1945. London: John Wisley \& Sons.

Diotallevi, L. (2017). Fine corsa. Bologna: EDB.

Fath, S. (2008). Dieu XXL. Paris : Autrement.

Foucault, M. (2009). Le courage de la verité. Paris : Seuil/Gallimard.

Freston P. (2002). Evangelical and Politics in Asia, Africa and Latin America. Cambridge: Cambridge University Press.

Hadaway, H. and Marler, P. (2005). How Many Americans Attend Worship Each Week? Journal for the Scientific Study of Religion, 44, pp. 307-322.

Hervieu-Léger, D. (1999). Le pelèrin et le converti. Paris : Flammarion.

Introvigne M. (1996). La sfida pentecostale. Torino: Elledici.

Kozinets, R. (2015). Netnography: Redefined: London: Sage.

Lehman, D. (2010). Rational Choice and the Sociology of Religion. In B.S. Turner (ed.), The New Blackwell Companion to the Sociology of Religion, London: Wiley-Blackwell, pp. 181-200.

Mariano, R., Gerardi A.D. (2019). Eleiçois presidenciais na America Latina em 2018 e ativismo politico. Revista USP, 120, pp. 61-78. 
Martin, D. (1990). Tongues of Fire. London: Blackwell.

Martin, D. (2002). Pentecostalism: The World Their Parish. London: Blackwell.

Meyer, B. (2009). Aesthetic Formations. New York: Springer.

Oro, A.P. (2020). Bolsonaro, gli evangelici e il coronavirus, Religioni e Società, 98, pp. 69-76.

Pace, E. and Butticci, A. (2010). Le religioni pentecostali. Roma: Carocci.

Pace, E. (2013). La comunicazione invisibile. Milano: San Paolo Edizioni.

Pace, E. (2017). Charisma as Transnational Enterprise. In P. Michel, A. Possamai, B.S. Turner (eds.), Religions, Nations, and Transnationalism in Multiple Modernities, London: Palgrave Macmillan, pp. 85-105.

Pace, E. (2018). Cristianesimo extra-large. Bologna: EDB.

Prandi, R., Dos Santos, W., Bonado, M. (2019). Igrejas evangélicas como maquina eleitorais no Brasil. Revista USP, 120, pp. 43-60.

Stark, R. \& Bainbridge, W.S. (1997). A Theory of Religion. New York: Peter Lang.

Thumma, S., Travis, D. \& Warren R. (2007). Beyond Megachurch Myth. San Francisco: Jossey-Bass.

Turner, V. (1982). From Ritual to Theatre. New York: PAJ.

Vries de, H. (2008). Religion: Beyond a Concept. New York: Fordham.

Young, L.A. (1997) (ed.). Rational Choice Theory and Religion. London: Routledge. 